\title{
Generation of energetic He atom beams by a pulsed positive corona discharge
}

\author{
Shui-Yin Lo a) \\ American Technologies Group, Inc., 1017 Mountain Avenue, Monrovia, California 91016 \\ Julio D. Lobo, Seth Blumberg, Theodore S. Dibble, ${ }^{b)} \mathrm{Xu} Z$ Zhang, ${ }^{\mathrm{c})}$ Chun-Cheng Tsao, \\ and Mitchio Okumura ${ }^{\mathrm{a}), \mathrm{d})}$ \\ Arthur Amos Noyes Laboratory of Chemical Physics, California Institute of Technology, Pasadena, \\ California 91125
}

(Received 7 October 1996; accepted for publication 28 January 1997)

\begin{abstract}
Time-of-flight measurements were made of neutral helium atom beams extracted from a repetitive, pulsed, positive-point corona discharge. Two strong neutral peaks, one fast and one slow, were observed, accompanied by a prompt photon peak and a fast ion peak. All peaks were correlated with the pulsing of the discharge. The two types of atoms appear to be formed by different mechanisms at different stages of the corona discharge. The fast atoms had energies of $190 \mathrm{eV}$ and were formed at the onset of the pulsing, approximately $0.7 \mu$ s before the maximum of the photon peak. The slow peak, composed of electronically metastable He atoms, originated 30-50 $\mu$ s after the photon pulse, and possessed a nearly thermal velocity distribution. The velocity distribution was typical of an undisturbed supersonic expansion with a stagnation temperature of $131 \mathrm{~K}$ and a speed ratio of 3.6. Peak intensities and velocities were measured as a function of source voltage, stagnation pressure, and skimmer voltage. (C) 1997 American Institute of Physics. [S0021-8979(97)04709-9]
\end{abstract}

\section{INTRODUCTION}

Corona discharges are a well known source of reactive ions, clusters, free radicals, and energetic species. For this reason, corona discharges and discharge-excited beam sources have been employed in a number of applications, including mass spectrometry, environmental remediation, ${ }^{1}$ laser spectroscopy, ${ }^{2,3}$ fast atom bombardment ionization, ${ }^{4}$ surface etching, ${ }^{5}$ and fundamental studies of hot atom chemistry and physics. ${ }^{6}$

One important application has been the generation of well defined beams of energetic atoms formed by corona and corona-excited discharges in pure inert gases. Searcy ${ }^{7}$ was the first to report the use of a combined sonic nozzle expansion and dc corona/glow discharge to generate helium metastables. Searcy's positive discharge generated a fast peak, which he assigned to photons, and a slow metastable peak. In 1975 Leasure et al., ${ }^{8}$ with a slightly modified design, observed two distributions from the spectrum: a fast, hot metastable peak with $5 \mathrm{eV}$ energy and a slower supersonic peak. By further modification of the source, Fahey et al. ${ }^{9}$ observed a fast $800 \mathrm{eV}$ helium peak, which they characterized as being predominantly ground state neutrals. In 1980, the same group ${ }^{10}$ using a negative discharge produced spectra containing only low-energy metastable and photon peaks, suggesting the fast neutrals were not generated with negative discharges. Verheijen et al. ${ }^{11}$ expanded on Fahey's work on negative discharges. Again, no fast peak was observed in their spectra. In their paper greater emphasis was given to the

\footnotetext{
a) Authors to whom correspondence should be addressed.

${ }^{b)}$ Present address: Department of Chemistry, SUNY College of Environmental Science and Forestry, Syracuse, NY 13210.

${ }^{c}$ Present address: International Rectifier Corp., 222 Kansas St., El Segundo, CA 90245.

${ }^{\mathrm{d})}$ Electronic mail: mo@cco.caltech.edu
}

intensity and velocity distributions of the slow metastable peak. The velocity distribution was found to agree with an undisturbed supersonic expansion model, and the intensity was found to correlate with the degree of excitation inside the source and the degree of de-excitation by collision with the residual gas. In 1991, Ferkel et al. ${ }^{12}$ used two negative discharges in series to produce a beam of metastable atoms in the $0.2-5 \mathrm{eV}$ range. Although Ferkel et al. focused on the slow metastable peak their spectra did show a small fast peak approximately $20 \mu$ s after the photon peak with translational energies around $120 \mathrm{eV}$.

Most sources used in applications of coronas for generation of energetic atom beams are based on Searcy's design, and involve discharges through a dielectric nozzle separating high and low pressure regions. The discharge occurs from the point of a sharp electrode in the high pressure region confined by the dielectric (e.g., a capped glass tube) to a skimmer located in vacuum $\left(10^{-2}-10^{-5}\right.$ Torr). The plasma undergoes a supersonic expansion through the nozzle, and the beam is extracted through the skimmer orifice.

Despite the widespread use of corona discharges for generating energetic beams, there has been little work oriented towards understanding the mechanism of fast neutral atom formation in these sources. On the other hand, many experiments concerning the properties of corona discharges have been conducted ${ }^{13-15}$ (for an insightful overview on mechanisms of coronas see $\mathrm{Loeb}^{16}$ ) and extensive theoretical literature exists regarding these discharges. ${ }^{13,17-19}$ However, the typical configuration for creating atom beams differs considerably from experiments on the properties of corona discharges. In the latter, discharges occur at high uniform pressures in ideal point-to-plane or point-to-cylinder electrode geometries. Furthermore, the currents in corona discharges are typically in the 10-100 $\mu \mathrm{A}$ range; in atom sources, the current between tip and skimmer may range from microam- 

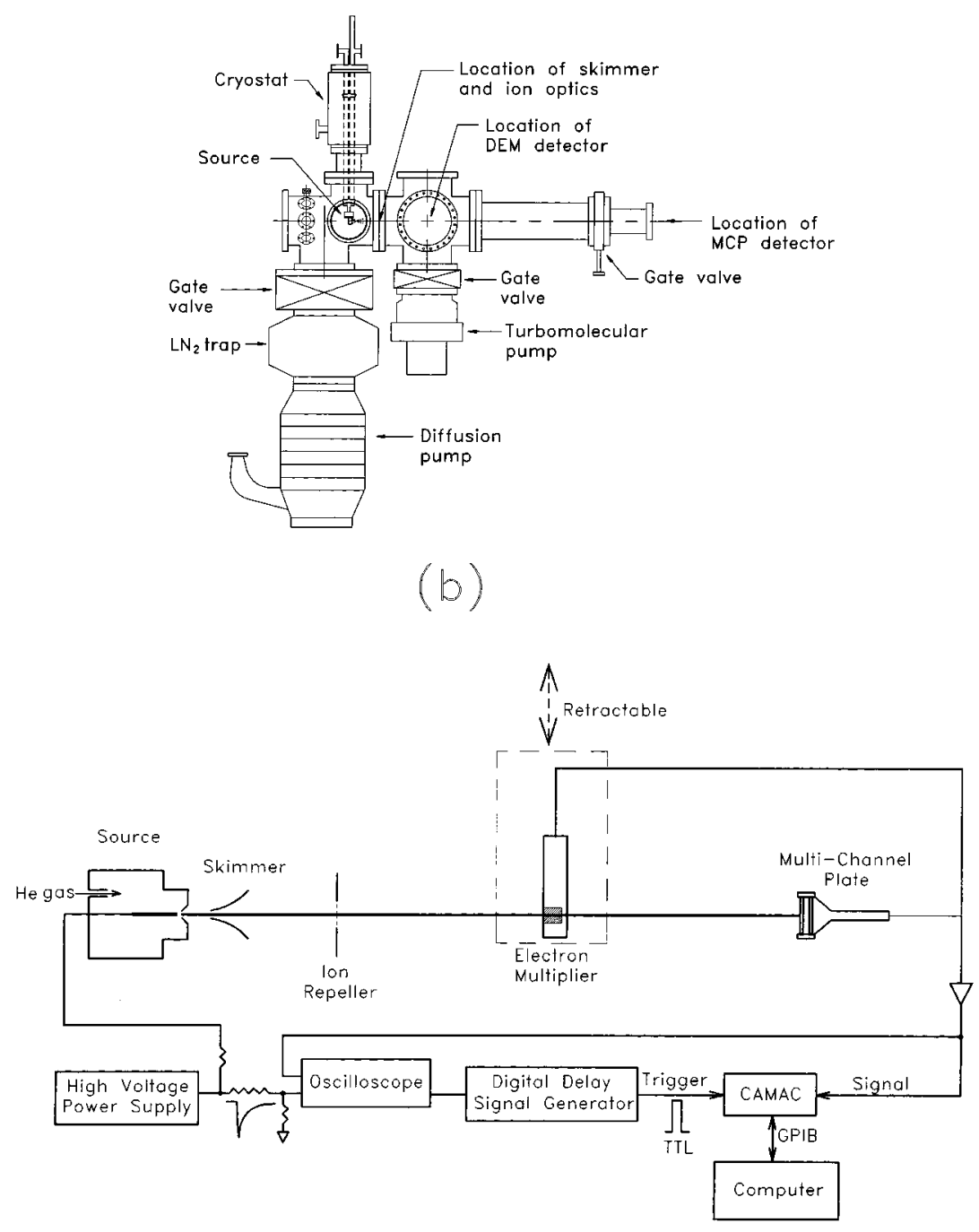

FIG. 1. (a) Assembling drawing of experimental apparatus. (b) Schematic of experiment including detection electronics.

peres to several milliamperes. Thus, it is not apparent to what extent the atom sources behave like conventional corona discharges.

There have been suggestions that the high velocity atoms generated by these atom sources are formed in the low pressure region, ${ }^{8}$ rather than within the high pressure discharge. Such arguments are based on the assumption that collisional relaxation of translationally fast atoms is rapid because the mean free path in the high pressure region is short $(100 \mathrm{~nm}$ length scale). In this picture, fast $\mathrm{He}$ atoms are formed by recombination of ions accelerated between the nozzle and skimmer. However, high velocities are also found within high pressure corona discharges, not as random motion of individual atoms but as bulk plasma waves. ${ }^{13-16}$ For example, at the onset of a pulsed positive corona discharge, ionization fronts (streamers) propagate rapidly from the anode tip to the cathode plane. The velocity of the ionization front is typically $10^{6}-10^{8} \mathrm{~cm} / \mathrm{s}$. Fast atoms can then be generated within a high pressure discharge if they are moving with the propagating plasma front. Several plausible mecha- nisms for the generation of fast atoms therefore exist.

In this article, we report a preliminary study of the energetic He atoms extracted directly through a small orifice in a pulsed point-to-plane corona discharge. Such sources have been used in the past to form cluster ions, but the properties of the neutral beams have not been reported. This work is part of an effort to develop a new source designed to generate $\mathrm{He}$ and $\mathrm{H}_{2}$ clusters and droplets at cryogenic temperatures. Here we characterize the fast and metastable atoms generated from within the high pressure discharge and we compare our results to energetic atom beams formed in standard (dielectric nozzle) sources. Based on the physics of corona discharges, we qualitatively discuss the mechanisms by which the fast atoms may be generated.

\section{APPARATUS}

The apparatus consisted of a cryogenically cooled, high pressure discharge source coupled to a time-of-flight (TOF) spectrometer. [A schematic is shown in Fig. 1(a).] Helium 
discharges were sampled through a small orifice in the source and expanded through two differentially pumped chambers: an expansion chamber and a detection chamber. TOF measurements were made in the detection chamber using two detectors located at different distances from the source.

The source was contained within a copper block mounted to the base of an RMC model CT-610-.1 liquid helium cryostat. In this experiment the cryostat was filled with liquid nitrogen. The discharge portion of the source, which was sealed with indium, consisted of a hollow cylin$\operatorname{der}(4 \mathrm{~mm}$ in length and $2 \mathrm{~mm}$ in radius) capped by a $25 \mu \mathrm{m}$ platinum nozzle. A 0.2 -mm-diam tungsten needle was fixed at $1 \mathrm{~mm}$ from the nozzle. The tip of the needle was electrochemically etched and inspected with a microscope before assembly. The needle was held by a screw in a macor plate which ensured perpendicular alignment and electrical insulation. A high voltage lead connected the needle to a $60 \mathrm{M} \Omega$ current-limiting resistor in series with an ammeter and high voltage power supply. A $200 \mathrm{M} \Omega-200 \mathrm{k} \Omega$ voltage divider was connected after the $60 \mathrm{M} \Omega$ ballast to allow monitoring of the discharge on an oscilloscope.

Before entering the source, helium gas of $99.995 \%$ purity passed through a $15 \mu \mathrm{m}$ filter, a liquid nitrogen cooled reservoir, and then made thermal contact with the cryostat. Prior to operation, the He inlet line was pumped to less than 100 mTorr lest water should be present. The helium pressure was controlled using a Matheson vacuum regulator (model 3491). As the gas flowed continuously into the source a point-to-plane corona discharge was struck between the charged needle and the nozzle. The plasma resulting from the discharge expanded through the aperture into the expansion chamber. This chamber was pumped by a 10 in. Varian diffusion pump baffled by a liquid nitrogen cryotrap. The base pressure in the expansion chamber was $2 \times 10^{-7}$ Torr, and rose to $7 \times 10^{-4}$ Torr when the inlet pressure reached about 800 Torr. Grounded liquid-nitrogen-cooled radiation shields enclosed the source to reduce stray electric fields and temperature loss. In addition, a grounded copper mesh wire was attached to the discharge cylinder also to reduce stray electric fields.

After traveling $2.5 \mathrm{~cm}$ from the nozzle in the expansion chamber, the beam was collimated by a skimmer with an aperture of $2.9 \mathrm{~mm}$ and a length of $2 \mathrm{~cm}$ before entering the detection chamber. The conical, nickel skimmer was mounted using a flat ring (less than $1 \mathrm{~mm}$ thick) clamp in order to prevent shock wave interference. It was electrically insulated and could be floated. Downstream $7 \mathrm{~cm}$ from the skimmer, a meshed plane aperture ion optic (repeller grid plate) was raised to a $2.2 \mathrm{kV}$ potential in order to repel positive ions. The detection chamber was pumped by a $1400 \ell / \mathrm{s}$ Pfeiffer turbomolecular pump. The base pressure in the detection chamber was $1.5 \times 10^{-7}$ Torr and rose to $1.4 \times 10^{-5}$ Torr at 800 Torr inlet pressure.

The apparatus for TOF measurements is shown schematically in Fig. 1(b). Absolute velocity measurements were made using the TOF spectra from two independent detectors: a Galileo microchannel plate (MCP) detector (model FTD2003) permanently situated $109.7 \mathrm{~cm}$ away from the nozzle, and a direct dynode electron multiplier (DEM) detector (model AF820 from ETP Ltd.) situated on a retractable mount $32.5 \mathrm{~cm}$ from the source. Care was taken to ensure reproducibility of the DEM alignment. A grounded mesh was fixed $0.2 \mathrm{~cm}$ before the MCP to avoid acceleration of ions. The voltage at the MCP cathode could be adjusted up to $-2.0 \mathrm{kV}$ and the anode was typically set at $-100 \mathrm{~V}$. The cathode of the DEM was raised to $-1390 \mathrm{~V}$.

The signals from the detectors were amplified $(\times 25)$ and then digitized by a Le Croy TR8818A transient recorder. A Le Croy 6010 Magic Controller communicated via a GPIB interface to a PC where data were recorded. Sampling rates varied from $10 \mathrm{~ns} /$ point to $640 \mathrm{~ns} /$ point. The trigger signal of the TOF was either taken from the sharp falling edge of the discharge voltage or, as was later found more appropriate, from the signal itself. The trigger signal was fed first to a Tektronix oscilloscope (model 2465A), because of its higher trigger sensitivity. A synchronous peak from the oscilloscope was then fed to a digital delay generator (model DG-535 from Stanford Research Systems) which generated a TTL trigger pulse for the transient recorder.

\section{RESULTS}

The source discharge exhibited pulsing behavior for a range of source voltages (both positive and negative) and source pressures. Discharge periods were on the order of milliseconds and varied with the source conditions. For the base condition of needle voltage $V_{\text {source }}=2.0 \mathrm{kV}$ and source inlet pressure $p=660$ Torr, the period was $5.3 \mathrm{~ms}$. The period was found to vary inversely with source voltage, increase with source pressure, and increase as the skimmer voltage was raised from negative to positive values. At pressures lower than 600 Torr, we found that the periodic discharge behavior was unstable. At these conditions, it was often necessary to initiate the discharge by temporarily increasing the pressure, or by increasing the voltage.

Due to stray capacitance and impedance mismatch, we were not able to accurately measure the fall time of the discharge, but an upper limit of $10 \mu$ s was established for positive discharges (needle as anode). Within this time the voltage dropped at least $40 \%$ of the maximum value. The discharge voltage then remained at this low level for 10-20 $\mu$ s, before slowly rising back to the initial voltage. Measurements of the voltage divider signal indicated that despite the high source voltage (defined as the voltage at the power supply) in the range from 1 to $4.8 \mathrm{kV}$, the actual potential across the needle ranged approximately from 300 to $450 \mathrm{~V}$. The average current through the source increased with the source voltage, and was in the range from 15 to $85 \mu \mathrm{A}$. Over a period of the discharge oscillation, the current changed by a few microamps.

The detector signal was composed of ionic and neutral peaks (Fig. 2) correlated temporally to the fast drop in the source voltage. By increasing the voltage on the ion repelling grid, we were able to eliminate the ion contribution and isolate the neutral component, except for a small ionic peak easily identified by its dependence on the repeller plate voltage and skimmer voltage. 


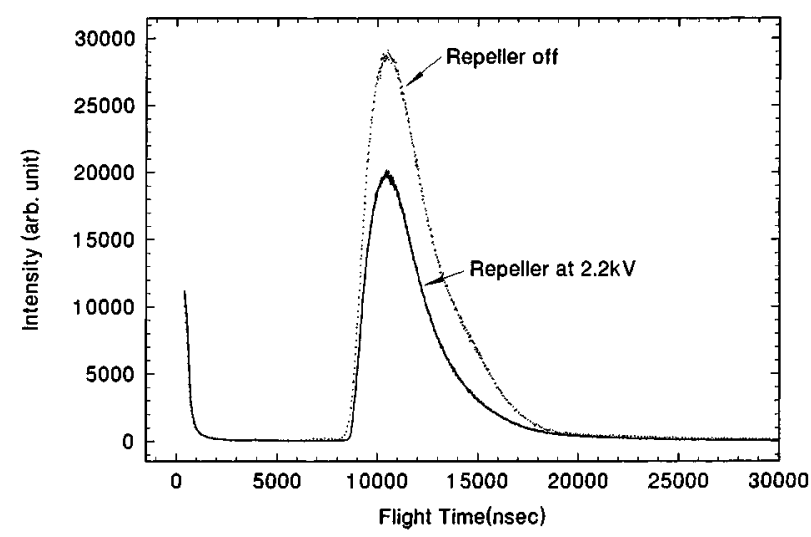

FIG. 2. TOF spectra of fast neutral peak with and without repelling potential at conditions of $V_{\text {source }}=2.0 \mathrm{kV}, p=660$ Torr, and $V_{\text {skimmer }}=-16 \mathrm{~V}$. The spectrum with repeller off includes signal due to ions, and is thus larger than the spectrum with the repeller at $2.2 \mathrm{kV}$, where the ions are eliminated.

For positive discharges, the neutral TOF spectra contained three large peaks (Fig. 3). The earliest peak arrived within $1 \mu$ s of the discharge onset. When the pressure in the detection chamber was raised to $5 \times 10^{-4}$ Torr, the signal from the two slower peaks decreased but the very fast signal remained unchanged. This observation indicated that the fast peak was generated by photons, which would have been unaffected by the higher background gas pressure. We assigned the remaining two peaks, which arrived at approximately 10 and $300 \mu \mathrm{s}$ at the farther MCP detector, to neutral He atoms.

The velocities of the neutral atoms were measured independent of the discharge trigger by comparing the arrival times at the two detectors. For a typical run, we found that the two neutral atom peaks typically corresponded to velocities of $\approx 9.6 \times 10^{4}$ and $\approx 1.2 \times 10^{3} \mathrm{~m} / \mathrm{s}$ (He atoms with kinetic energies of 190 and $0.03 \mathrm{eV}$, respectively). We inferred that the slowest peak consisted of electronically metastable helium atoms, since the detectors were only sensitive to par- ticles having more than $20 \mathrm{eV}$ in energy. With the current apparatus we were unable to determine if the faster peak was composed of ground state atoms, metastable atoms, or both. The integrated intensities of the slow and fast peaks were approximately the same. However, we do not know the relative sensitivities of the detectors to translationally energetic atoms versus electronically metastable atoms; therefore, we could not quantitatively estimate the relative fluxes of the two types of atoms.

From the velocity measurements and the known nozzledetector distances, we were able to determine the time of origin (time zero) of the fast and slow peaks observed. We found that the fast peak originated at the leading edge of the photon peak, $\approx 0.7 \mu$ s before the photon maximum. In contrast, the slow metastable peak originated $\approx 40 \mu$ s after the photon peak. The dual detector setup also allowed us to compare the velocity distributions at both positions. Upon scaling the fast peak signal obtained at the two different detectors, we observed that the distribution at the MCP (rear) detector was $\approx 20 \%$ broader than the distribution at the DEM (front) detector. This result implied a small dispersion term in addition to a simple velocity distribution. There are two possible explanations: some neutrals were generated in the nozzleskimmer region after they had expanded out of the source, or, there was a small spread in time of origin, with the atoms generated first having slower speed than the atoms generated later. The same scaling comparison for the slow peaks showed that the distribution at the MCP was broader by only $3 \%$ than that of the DEM. Neither the fast nor the slow metastable velocity distributions fit to a MaxwellBoltzmann distribution. However, except for a high velocity tail, the slower peak could be fit to the expression: $f(\nu)$ $=A(m / 2 k T)^{3 / 2} \nu^{3} \exp \left[-(m / 2 k T)\left(\nu-\nu_{0}\right)^{2}\right]$. This formula is appropriate for the velocity distribution of a gas undergoing supersonic expansion. The fit was characterized by a thermal temperature of about $21 \mathrm{~K}$ and a mean velocity $\nu_{0}$ of $\approx 1067$ $\mathrm{m} / \mathrm{s}$. Therefore, the slow peak had a speed ratio of about 3.6.

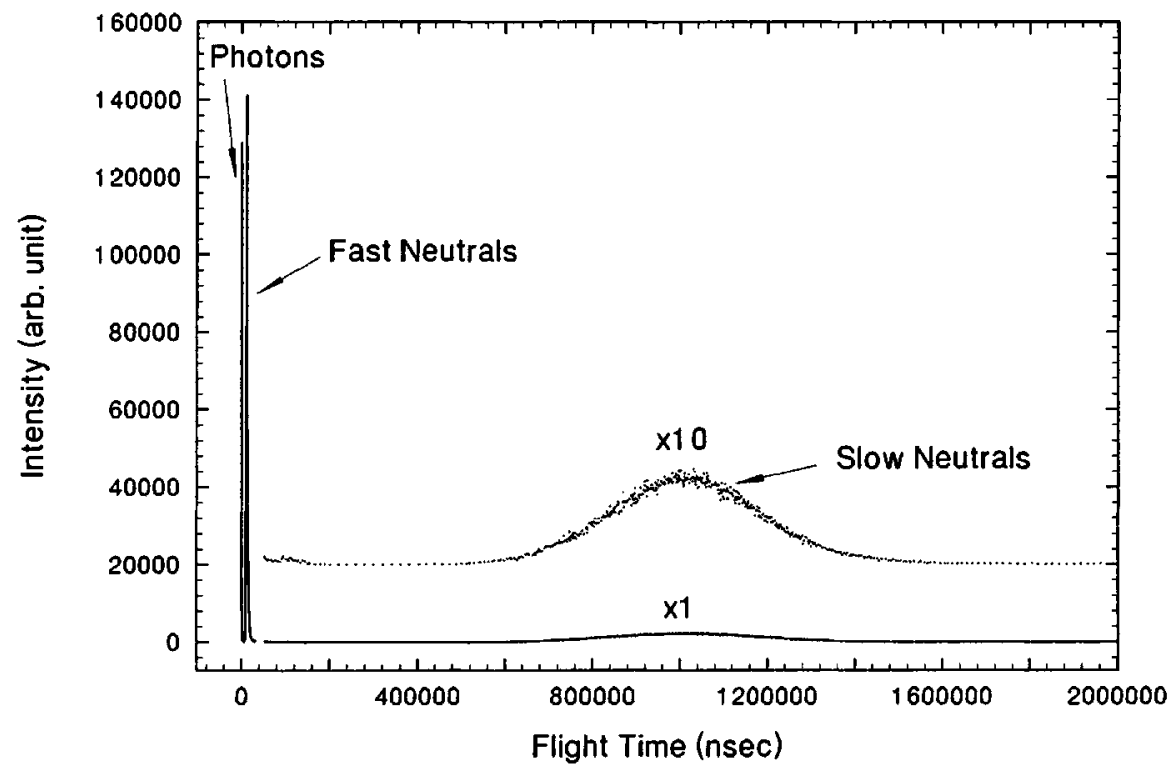

FIG. 3. TOF spectrum showing photon peak, fast neutral peak and slow neutral peak. Slow neutral peak is also scaled up by a factor of 10 for better viewing. 
(a)

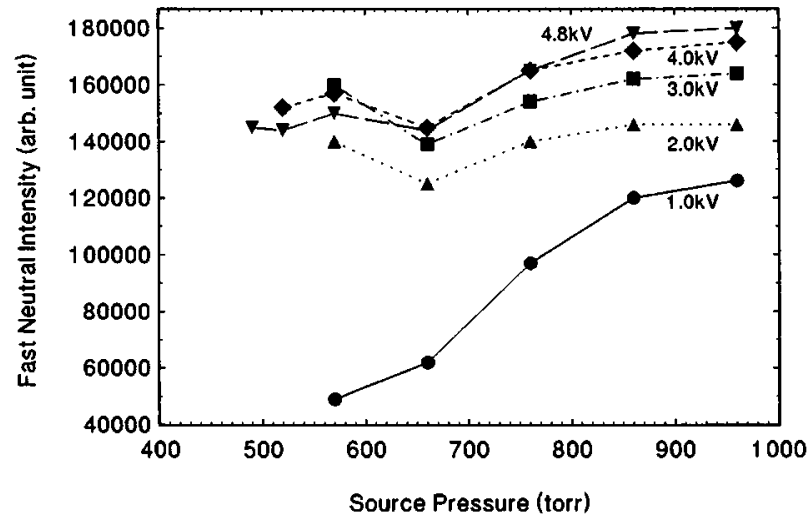

(b)

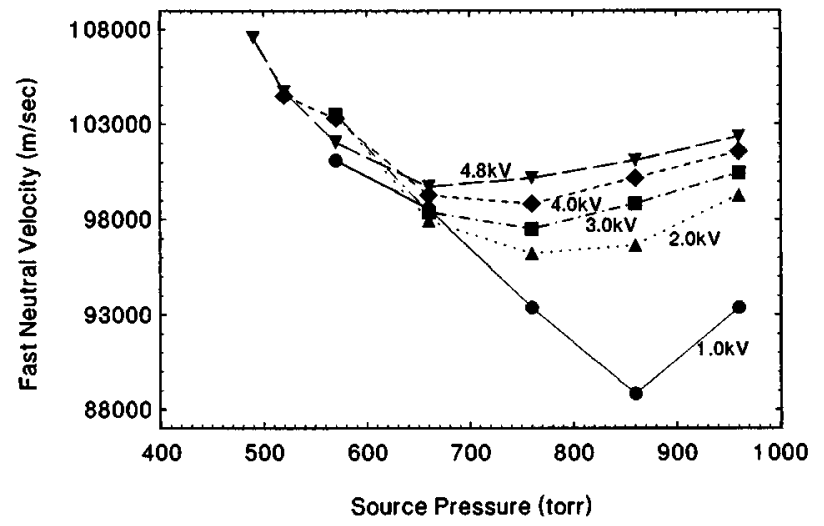

FIG. 4. (a) Dependence of fast neutral intensity on source pressure and voltage. (b) Dependence of fast neutral velocity on source pressure and voltage.

In contrast, the velocity distribution of the fast peak had a characteristic long tail on the slow velocity edge and sharp edge on the high velocity end.

For the fast peak, the dependencies of peak velocity and intensity on source voltage and inlet pressure were studied in the ranges $V_{\text {source }}=1.0-4.8 \mathrm{kV}$, and $p=460-970$ Torr [Figs. 4(a) and 4(b)]. In general, we found that the source voltage and pressure dependencies were coupled. For the fast peak, at pressures between 660 and 960 Torr, the intensity increased by $40 \%-100 \%$ as the source voltage went from 1 to $4.8 \mathrm{kV}$. At 570 Torr, the intensity tripled as source voltage went from 1 to $3 \mathrm{kV}$, but then decreased by a few percent as the source voltage reached $4.8 \mathrm{kV}$. TOF spectra showing the dependence on source voltage are presented in Fig. 5. In contrast to the large changes in intensity, only small changes of velocity were observed for the fast peak when the source voltage was varied, although the trends were similar. From 760 to 960 Torr, the velocity increased by at most $10 \%$ as the source voltage went from 1 to $4.8 \mathrm{kV}$. At 570 Torr, the velocity increased by $3 \%$ for a source voltage range of 1 to 3 $\mathrm{kV}$, and then decreased by $2 \%$ as the source voltage went from 4 to $4.8 \mathrm{kV}$. The intensity and velocity of the fast peak exhibited a weaker dependence on the stagnation pressure setting than on source voltage. At $1 \mathrm{kV}$, the intensity increased by $100 \%$ as the pressure was increase from 670 to

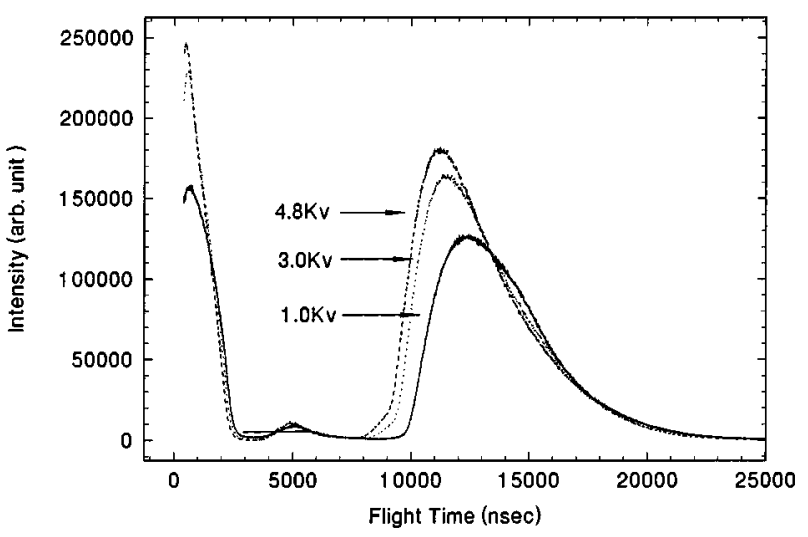

FIG. 5. TOF spectra of fast atom peak for various source voltages at conditions of $p=960$ Torr and $I_{\text {skimmer }}=-16 \mathrm{~V}$.

970 Torr. At higher voltages, the intensity increased no more than $10 \%$. The dependence of intensity on pressure was unclear for pressures below 670 Torr. The velocity dependence went through a minimum at 650-850 Torr when the pressure was varied from 470 to 970 Torr, but the variation in the velocity was again very small ( $<10 \%$ typically).

The dependencies of the intensity and velocity of the slow peak on source voltage and pressure were also studied for the same conditions mentioned above [Figs. 6(a) and $6(b)]$. The dependence of intensity on voltage and pressure was opposite to that observed for the fast peak. The intensity was found to decrease as much as $50 \%$ with rising voltage and decrease as much as $80 \%$ with increasing pressure. The velocity, which showed little variation, increased $5 \%$ with increasing voltage and $10 \%$ with increasing pressure.

The dependence on skimmer voltage for the fast peak was measured in the range $V_{\text {skimmer }}=-400$ to $+300 \mathrm{~V}$ [Figs. 7(a) and 7(b)]. A characteristic jump in intensity and velocity were observed as the polarity was switched. The intensity changed by less than $10 \%$ for voltages from -400 to -100 $\mathrm{V}$, but it decreased by $1000 \%$ near $0 \mathrm{~V}$. From +100 to +300 $\mathrm{V}$ it only decreased by an additional $50 \%$. The velocity did not change for low skimmer voltages, but increased by about $8 \%$ as the skimmer voltage increased from -200 to $100 \mathrm{~V}$. Above $100 \mathrm{~V}$, the velocity was constant. We also observed a qualitative change in the shape of the fast peak as the skimmer voltage was changed from 0 to $-400 \mathrm{~V}$. A second, faster component $\left(1.2 \times 10^{5} \mathrm{~m} / \mathrm{s}, 300 \mathrm{eV}\right)$ appeared to augment in magnitude with higher negative skimmer voltages (Fig. 8). We saw a distinct glow protruding from the nozzle when the skimmer was at very negative voltages ( $V_{\text {skimmer }}$ $<-300 \mathrm{~V}$ ); the plasma glow appeared to extend further into the source region.

For the slow peak, the intensity also showed a large change as the skimmer polarity was switched [Fig. 7(a)]. From -400 to $-100 \mathrm{~V}$ the intensity increased by $10 \%$, from -100 to $+100 \mathrm{~V}$ it decreased by $52 \%$, and then from 100 to $300 \mathrm{~V}$ it increased by less than $20 \%$. The skimmer voltage had a negligible effect on the velocity of the slow peak.

For negative discharges (needle as cathode), only the photon and slow metastable peak were observed. These were not characterized further in the current experiment. 
(a)

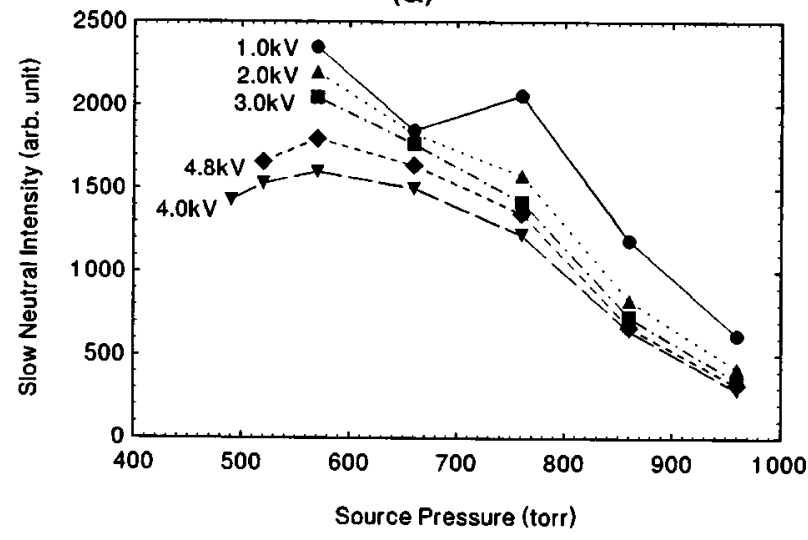

(b)

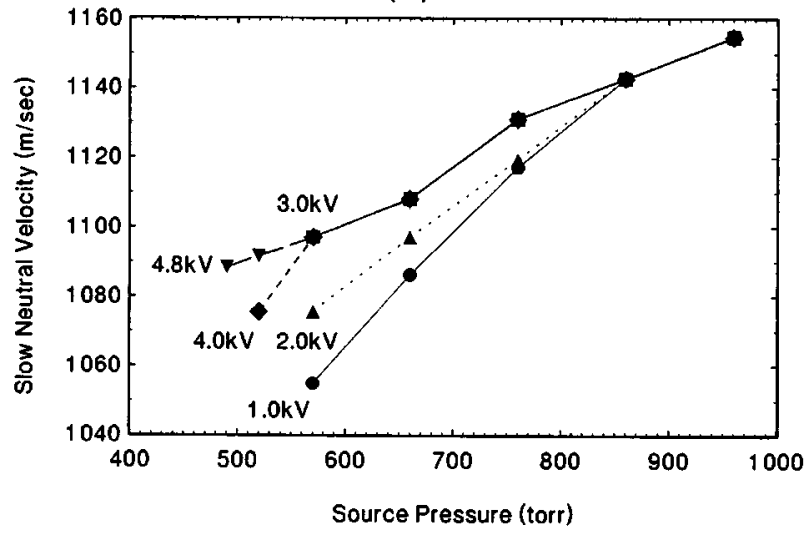

FIG. 6. (a) Dependence of slow neutral intensity on source pressure and voltage. (b) Dependence of slow neutral velocity on source pressure and voltage.

\section{DISCUSSION}

\section{A. Corona discharge}

Although the conditions (1 mm gap, $77 \mathrm{~K}$, nonattaching gas) are somewhat different from conventional air corona discharges, the discharge we observe in this experiment exhibits behavior similar to other repetitive, pulsed, positive point corona discharges. Such discharges are characterized by an initial ionizing streamer which occurs within the first several nanoseconds of the breakdown. Photoionization is often thought to be a major contributor to ionization processes during streamer propagation. If so, emission of very short wavelength photons should be a characteristic of streamers, and would account for some of the early photons we observe. The primary streamer may be followed by secondary streamers and the formation of a longer-lived plasma similar to a glow discharge. The UV photon burst lasts for only $\approx 1 \mu \mathrm{s}$, but our current measurements indicate that the discharge persists for about $30-50 \mu$ s before slowly relaxing.

\section{B. Translationally hot atoms}

There are several possible mechanisms for the formation of the fast He atoms ( $\nu \approx 10^{5} \mathrm{~m} / \mathrm{s}$ and $E \approx 190 \mathrm{eV}$ ). These atoms originate within $1 \mu$ s of the onset of the discharge. (a)

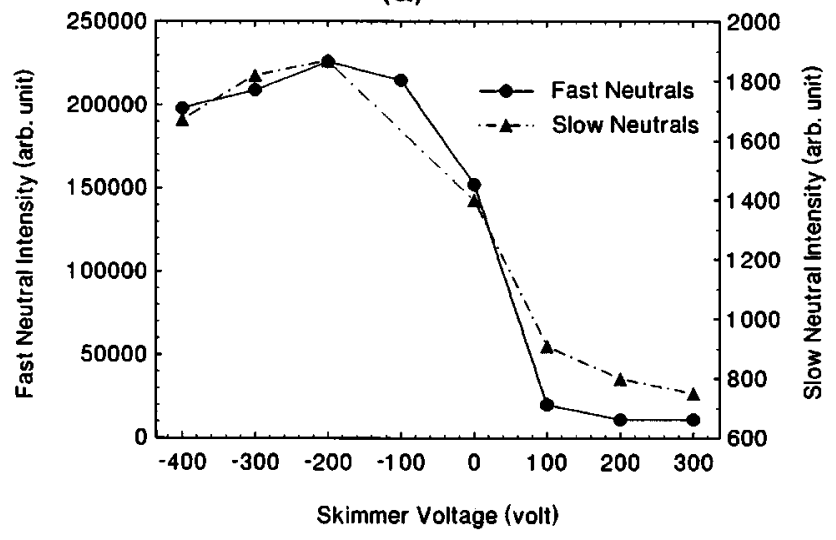

(b)

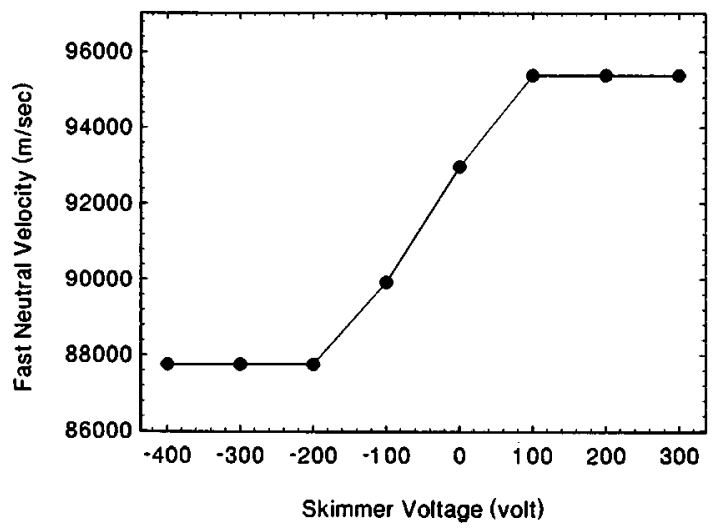

FIG. 7. (a) Dependence of fast and slow neutral intensities on skimmer voltage $\left(V_{\text {source }}=2.0 \mathrm{kV}, p=660\right.$ Torr). (b) Dependence of fast neutral velocity on skimmer voltage. (The slow neutral velocity was independent of skimmer voltage.)

From this result we infer that the translationally hot atoms are associated with the early breakdown of the gas. Furthermore, the similarity in velocity distributions and intensities between the fast atoms and the ion peaks [observed with the

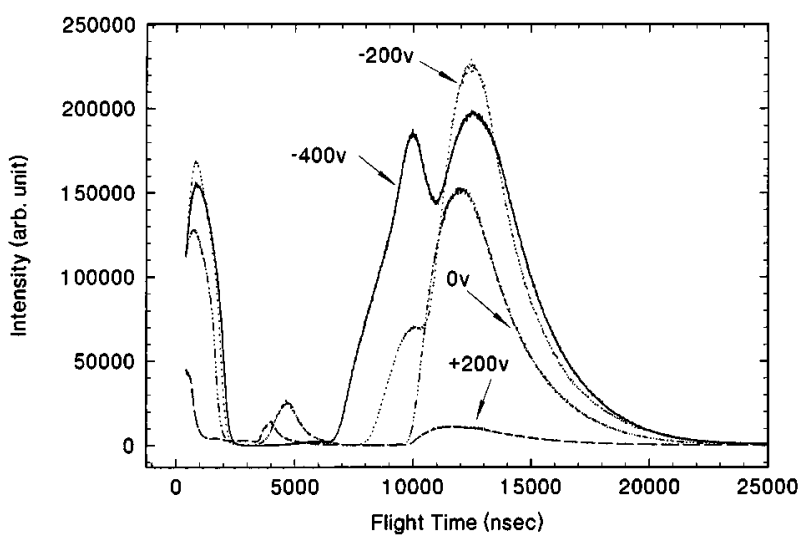

FIG. 8. TOF spectra of fast neutral atoms at various skimmer voltages showing the photon peak, an ionic pulse which increases as the skimmer voltage is varied from +200 to $-400 \mathrm{~V}$, and the fast neutral peak ( $V_{\text {source }}$ $=2.0 \mathrm{kV}, p=660$ Torr $)$. 
detector voltage turned on and off (see Fig. 2)] suggest that the fast atoms and the ions have a common origin.

The early time of origin of the fast atoms, $\approx 0.7 \mu$ s before the maximum in the photon peak, should coincide with streamer formation. If this is the case, these fast atoms and ions may be formed in the primary streamer itself. This notion is supported by the similarity between the velocities we observe for the fast atoms (and ions) and the propagation velocities of streamers observed directly by optical methods in pulsed corona discharges. For example, Grangé et al. ${ }^{13}$ observed ionizing front velocities of $2 \times 10^{5} \mathrm{~m} / \mathrm{s}$. Their result is within a factor of 2 of the fast helium velocities we measure; however, we cannot make a direct comparison, since Grangé's experiments were performed in air with a $1 \mathrm{~cm}$ gap at a $7 \mathrm{kV}$ applied potential.

Alternatively, the similarity between the fast atom energy $(\approx 190 \mathrm{eV})$ and the transient discharge voltage (minimum at $150 \mathrm{~V}$ and maximum at $400 \mathrm{~V}$ ) suggests that the fast atoms may be formed by recombination of ions accelerated in the cathode fall region. Neutralization could occur as the ions pass through the orifice in the cathode, or later as it expands. This picture might also be compatible with streamer ionization. Once the streamer reaches a point where it interacts with the cathode space charge, ions at the head of the streamer would be accelerated towards the cathode, and thus be emitted with high energies.

A third alternative, electrohydrodynamic acceleration of the background gas, does not seem plausible. Eichwald et $a l .{ }^{19}$ have calculated the velocity field induced in the neutral background for short gap, highly repetitive He discharges. They found maximum velocities on the order of $10 \mathrm{~m} / \mathrm{s}$, well below the velocities of the fast atoms emitted by this source.

\section{Metastable atoms}

The slower metastable atoms appear to have a significantly different origin from that of the translationally excited atoms. These atoms originate 30-50 $\mu$ s after the photon peak, indicating that they are formed in the later glowdischarge portion of the pulsed discharge sequence. Furthermore, the intensities of the slow metastable peaks generally have the opposite dependence on source conditions relative to the fast peaks. The slow metastable velocities are also closer to thermal velocities, with a distribution characteristic of atomic beams generated by a supersonic expansion through a small orifice rather than acceleration by high fields. Thus, we attribute the slow peak to metastable atoms that are emitted from a plasma with thermalized neutral gas background, in agreement with the metastables observed by others. ${ }^{7,8,10-12}$

If we assume an isentropic expansion, then we find that the metastable atoms are produced from expansion of a gas with a stagnation temperature of $131 \mathrm{~K}$. Thus, the plasma 30-50 $\mu$ s after initial breakdown has a neutral background temperature approximately double that of the cell walls. Plasma effects could also explain the high velocity tail of the distribution, which exceeds that expected for a supersonic distribution, since collisions with more energetic charged particles would lead to excess population in high translational energies.
Based on a supersonic expansion model, the dependencies of the slow peak on source pressure and voltage can be qualitatively understood. Higher pressures lead to a stronger expansion from the nozzle, resulting in faster velocities. The stronger expansion also increases the flux and thus the intensity. However, the higher stagnation density should also lead to increased quenching of the metastable species by collision prior to expansion. The decrease in metastable intensity observed implies that the quenching process dominates. The weak dependence of the intensity and velocity on voltage suggests that the stagnation temperature and the local concentration of excited helium atoms in the glow-discharge region are relatively unchanged. Changes in the electrode voltage may be screened by the plasma.

\section{Comparison with previous beam experiments}

Both translationally hot and thermal metastable atoms have been observed previously. All of these earlier studies used sources with dielectric nozzles in which the discharge was struck between a point inside the nozzle and the skimmer. Curiously, no paper reported photons, fast atoms, and thermal metastables simultaneously. Several papers ${ }^{7,8,10-12}$ have described thermal metastable beams from corona discharge sources, both pulsed and continuous. These authors arrived at a similar conclusion for the mechanism of metastable atom beam generation, i.e., supersonic expansion from a high pressure plasma. The origin of the fast atoms, however, was never addressed.

Fast atoms have been detected with energies ranging from 5 to $800 \mathrm{eV}$ in pulsed and continuous discharges. Although Searcy et ll $^{7}$ assigned a fast neutral peak to photons, Leasure et $a l .{ }^{8}$ speculated that the first group might have mistakenly attributed a fast peak to photons. Without knowledge of the time resolution in Searcy experiment it is impossible to know if they observed photons, a fast peak, or both. Leasure et al. observed helium atoms at $5 \mathrm{eV}$ and attributed them to metastables. The authors conjectured that these fast atoms were accelerated by collisions in the low pressure region (outside the nozzle). Their reasoning was based on the assumption that metastables accelerated before the nozzle would probably be quenched due to the high density in the stagnation region. Fahey et al., ${ }^{9}$ in their 1978 publication, observed an $800 \mathrm{eV}$ neutral peak. They detected neither a photon peak nor any other peak with energies below $100 \mathrm{eV}$. The significantly higher energies may be attributable to different source conditions (8-9 $\mathrm{kV}$ and order of milliamps discharge currents), but there is currently no model to account for this result.

Although the anode in our experiment is the grounded nozzle, the skimmer voltage does produce strong effects on the plasma. We found that the skimmer voltage has a significant effect on the intensity of the fast atom peak. Application of a high positive potential leads to a large decrease in neutral intensity (Fig. 8). This result suggests that the positive field of the charged skimmer (which was located $2.5 \mathrm{~cm}$ from the nozzle) might have been sufficient to affect the plasma at the source aperture. Alternatively, if most of the neutralization indeed occurs in the low pressure region, then a positive bias on the skimmer may repel the ions before 
neutralization. By applying a large negative potential to the skimmer, an increase in plasma glow brightness is observed outside of the nozzle. Thus, the plasma appears to be drawn further out of the source. This correlates with the large secondary peak arriving at $\approx 10 \mu$ s (with energy equivalent to $\approx 300 \mathrm{eV}$ ) in Fig. 8. It is conceivable then that the $800 \mathrm{eV}$ atoms observed by Fahey et al. ${ }^{9}$ were formed either in a secondary discharge or by single collision processes in the nozzle-skimmer region. Further experiments are needed to clarify the role of the low pressure region in fast atom formation.

\section{E. Hypothetical mechanism}

As discussed above, these observations can be explained by conventional corona discharge theory which assumes space-charge limited ion densities. However, one of the authors ${ }^{20}$ has proposed another possible mechanism based on the assumption that a strongly coupled plasma is formed. ${ }^{21}$ This mechanism requires the strong assumption that all helium atoms are ionized in a region close to a sharp needle tip ( $r_{0} \ll 1 \mu \mathrm{m}$ ), thus creating locally high ion densities. As the ions move towards the nozzle, they are cooled by the neutral background. They may then form a strongly coupled plasma $^{22}$ with a coupling given by $\Gamma=\left(e^{2} / a_{i}\right) / k T$, where $T$ is the temperature of the ions, and $a_{i}$ is the distance between ions. The density of ions varies as $1 / r^{2}$, where $r$ is the distance from the tip of the needle. At the glow region, if complete ionization led to a density of $2 \times 10^{9} \mathrm{~cm}^{-3}$, then at $r \sim 100 r_{0}$, the density would be $2 \times 10^{15} \mathrm{~cm}^{-3}$. At $T=150$ $\mathrm{K}$, this gives a coupling strength of $\Gamma \approx 1$, which is the nominal condition for a strongly coupled plasma behaving like a liquid. ${ }^{21}$ As the streamer develops from the needle the electric field will propagate the plasma towards the cathode. If the plasma carries the neutral atoms with it, the neutrals will be accelerated together with the ions. This mechanism may be consistent with our results. For fast neutrals higher applied voltages will result in higher velocity until a terminal velocity is reached. Higher applied voltage also means higher current, and hence higher intensity. A variation of pressure also will vary the viscosity of the gas, and there is a nonlinear dependence on pressure. For slow neutrals when the liquid plasma moves forward, there is an angular velocity. ${ }^{23}$ The higher applied voltage will result in a higher angular velocity and ions will reach a larger volume of neutrals. This effect increases ion cooling and results in a lower temperature of the average residue ions. These ions recombine with electrons to form the metastable helium. Since the ions are cooled by a larger volume of cold gas the ion density is reduced, thus a lower intensity of slow metastable neutrals is produced. Numerical simulations must be performed to test these conjectures, since this is an alternative to the more conventional explanations that require only spacecharged limited density.

\section{CONCLUSIONS}

In our experiment, translationally energetic and slow metastable neutral atoms were observed from a repetitive, pulsed corona discharge, accompanied by photon and ion peaks. The atoms, ions, and photons correlated temporally with the pulsing of the discharge. The fast atoms are associated with the onset of the discharge, at the leading edge of the photon pulse, and may result from the initial streamer in the discharge. The slow metastable peak had a velocity distribution typical of an undisturbed supersonic expansion, with a stagnation temperature of $131 \mathrm{~K}$, and originated 30-50 $\mu$ s after the photon pulse.

There are many possible directions for further investigation. First, experiments are needed to establish a more direct correlation of the discharge plasma phenomena with fast atoms. Perhaps the most direct method would be to obtain images of streamers within a typical corona discharge source and compare the timing and velocity of the streamer with that of the fast atoms. A complementary approach would be to relate the time dependence of the discharge current with the time of origin of the fast atoms. Second, a more comprehensive study of source conditions, especially the needle-tonozzle gap, voltage and current, stagnation temperature, and skimmer is necessary to account for the wide variations in fast atom velocities and to provide a means of accurately controlling the beam velocity. Finally, control over beam energy could lead to the development of a very powerful new tool with many possible applications. This source could be used as an alternative to other hyperthermal neutral beam sources. For example, the corona source may prove to be simpler, more compact, and more versatile than the laserdetonation sources currently being developed for applications in semiconductor etching. ${ }^{24}$

\section{ACKNOWLEDGMENTS}

This work was performed at the California Institute of Technology with funding provided by American Technologies Group, Inc. The authors wish to thank Dr. Young K. Bae for helpful advice.

${ }^{1}$ T. H. Teich and M. Jacob, Inst. Phys. Conf. Ser. 143, 227 (1995).

${ }^{2}$ P. C. Engelking, Rev. Sci. Instrum. 57, 2274 (1986).

${ }^{3}$ M. E. Derose, P. G. Carrick, and C. R. Brazier, J. Mol. Spectrosc. 174, 379 (1995).

${ }^{4}$ D. Faubert, G. J. C. Paul, J. Giroux, and M. J. Bertrand, Int. J. Mass Spectrom. Ion Processes 124, 69 (1993).

${ }^{5}$ T. Nishimura, H. Suzuki, and T. Satake, Appl. Surf. Sci. 70, 133 (1993).

${ }^{6}$ U. Buck, in Atomic and Molecular Beam Methods, edited by G. Scoles (Oxford University Press, Oxford, 1988).

${ }^{7}$ J. Q. Searcy, Rev. Sci. Instrum. 45, 589 (1974).

${ }^{8}$ E. L. Leasure, C. R. Mueller, and T. Y. Ridley, Rev. Sci. Instrum. 46, 635 (1975).

${ }^{9}$ D. W. Fahey, L. D. Schearer, and W. F. Parks, Rev. Sci. Instrum. 49, 503 (1978).

${ }^{10}$ D. W. Fahey, W. F. Parks, and L. D. Schearer, J. Phys. E 13, 381 (1980).

${ }^{11}$ M. J. Verheijen, H. C. W. Beijerinck, L. H. A. M. v. Moll, J. Driessen, and N. F. Verster, J. Phys. E 17, 904 (1984).

${ }^{12}$ H. Ferkel, R. Feltgen, and D. Pikorz, Rev. Sci. Instrum. 62, 2626 (1991).

${ }^{13}$ F. Grange, N. Soulem, J. F. Loiseau, and N. Spyrou, J. Phys. D 28, 1619 (1995).

${ }^{14}$ E. Marode, J. Appl. Phys. 46, 2005 (1975).

${ }^{15}$ G. F. L. Ferreira, O. N. Oliveira, Jr., and J. A. Giacometti, J. Appl. Phys. 59, 3045 (1986). 
${ }^{16}$ L. B. Loeb, Electrical Coronas Their Basic Physical Mechanism (University of California Press, Berkeley, 1965).

${ }^{17}$ P. Bayle and B. Cornebois, Phys. Rev. D 31, 1046 (1985).

${ }^{18}$ N. Y. Babaeva and G. V. Naidis, Phys. Lett. A 215, 187 (1996).

${ }^{19}$ O. Eichwald, M. Jugroot, P. Bayle, and M. Yousfi, J. Appl. Phys. 80, 694 (1996).

${ }^{20}$ S. Y. Lo (unpublished).
${ }^{21}$ S. Ichimaru, Rev. Mod. Phys. 65, 255 (1993).

${ }^{22}$ See, for example, R. C. Davidson, Physics of Nonneutral Plasmas (Addison-Wesley, Reading, MA, 1990), pp. 65-75.

${ }^{23}$ E. M. Lifshitz and L. P. Pistaevskii, Physical Kinetics (ButterworthHeinemann, Oxford, 1981), pp. 63-71.

${ }^{24}$ K. P. Giapis, T. A. Moore, and T. K. Minton, J. Vac. Sci. Technol. A 13, 959 (1995). 